\title{
The Dark Side of Nrf2 in the Heart
}

\author{
Huimei Zang ${ }^{1}$, Roy Oomen Mathew² and Taixing Cui ${ }^{\text {* }}$ \\ ${ }^{1}$ Department of Cell Biology and Anatomy, University of South Carolina School of Medicine, Columbia, SC, United States, \\ ${ }^{2}$ Division of Nephrology, Department of Medicine, Columbia VA Healthcare System, Columbia, SC, United States
}

OPEN ACCESS

Edited by:

Xuejun Wang,

University of South Dakota,

United States

Reviewed by:

Jiliang Zhou,

Georgia Health Sciences University,

United States

Rajasekaran

Namakkal-Soorappan,

University of Alabama at Birmingham,

United States

${ }^{*}$ Correspondence:

Taixing Cui

taixing.cui@uscmed.sc.edu

Specialty section

This article was submitted to

Striated Muscle Physiology,

a section of the journal

Frontiers in Physiology

Received: 29 March 2020

Accepted: 02 June 2020

Published: 09 July 2020

Citation:

Zang $\mathrm{H}$, Mathew $\mathrm{RO}$ and Cui T

(2020) The Dark Side of Nrf2

in the Heart. Front. Physiol. 11:722.

doi: 10.3389/fphys.2020.00722
Nuclear factor-erythroid factor 2-related factor 2 (Nrf2) is a critical transcription factor that regulates the expression of over 1000 genes in the cell under normal and stressed conditions. These transcripts can be categorized into different groups with distinct functions, including antioxidative defense, detoxification, inflammatory responses, transcription factors, proteasomal and autophagic degradation, and metabolism. Nevertheless, Nrf2 has been historically considered as a crucial regulator of antioxidant defense to protect against various insult-induced organ damage and has evolved as a promising drug target for the treatment of human diseases, such as heart failure. However, burgeoning evidence has revealed a detrimental role of Nrf2 in cardiac pathological remodeling and dysfunction toward heart failure. In this mini-review, we outline recent advances in structural features of Nrf2 and regulation of Nrf2 activity and discuss the emerging dark side of Nrf2 in the heart as well as the potential mechanisms of Nrf2-mediated myocardial damage and dysfunction.

Keywords: Nrf2, oxidative stress, reductive stress, autophagy, heart failure

\section{INTRODUCTION}

Heart failure is defined as "a complex clinical syndrome that can result from any structural or functional cardiac disorder that impairs the ability of the ventricle to fill with or eject blood" (Hunt et al., 2005; Yancy et al., 2013). Recent studies have revealed that heart failure affects 1 in 5 Americans, and the prevalence and incidence of heart failure are still increasing (Yancy et al., 2013; Virani et al., 2020). Heart failure is usually the last stage of different cardiovascular diseases, such as hypertension that causes sustained pressure overload to the heart; coronary arterial diseases, leading to myocardial infarction or ischemia reperfusion-related cardiac injury; valvular disease, resulting in volume overload to the heart; and congenital heart disease. Despite the differential etiologies, the progression of heart failure goes through a common path; i.e., cardiac remodeling, which has been considered as epigenetic and genomic alterations as well as molecular and cellular responses, resulting in clinically manifested changes in geometry and function of the heart after cardiac pressure or volume overload and/or injury (Swynghedauw, 1999; Cohn et al., 2000; Heusch et al., 2014). Cardiac remodeling may be initially adaptive against various harmful insults but, when sustained, turns out to be maladaptive, or pathological, progressing to structural and functional changes that lead to heart failure. Cardiac maladaptive remodeling is usually characterized by myocardial hypertrophy, fibrosis, and cell death, which may be resulted from a complex interaction between cardiac myocytes and non-myocytes. The molecular mechanism of cardiac maladaptive remodeling that leads to heart failure in diverse pathological settings is poorly understood. The treatment of heart failure remains at a level of controlling symptoms and reducing risk factors without a cure. 
Oxidative stress, a state that occurs when the oxidative force, e.g., production of reactive oxygen species (ROS) exceeds the antioxidant capacity, is a common mechanism of various cardiac pathologies leading to heart failure (Tsutsui et al., 2011; Ahmed and Tang, 2012). However, several large clinical trials using antioxidant supplements, such as non-selective ROS scavenger vitamin $\mathrm{C}$, vitamin $\mathrm{E}$, and allopurinol, have found that non-specific scavenging of ROS does not help to prevent cardiovascular disease; instead, it may even be harmful (Li et al., 2009a; Ahmed and Tang, 2012). These studies suggest that effective therapeutic approaches for the treatment of cardiovascular disease may not be achieved without specific targeting the source of oxidative stress or the intrinsic antioxidant system. In this regard, nuclear factor-erythroid factor 2-related factor 2 (Nrf2), which has been historically considered as a master transcription factor of antioxidant defense, has evolved as an attracting therapeutic target for cardiovascular disease (Li et al., 2009a; Chen and Maltagliati, 2018; Ge et al., 2019). Despite the fact that Nrf2 appears to be a critical regulator of cellular defense against various pathological insults in the heart, burgeoning evidence has demonstrated a detrimental role of Nrf2 in cardiac disease progression (Cui et al., 2016). In this mini-review, we update recent advances in structural features and activity regulation of vertebrate $\mathrm{Nrf} 2$ and then have a close look on the dual effects of Nrf2 in the heart and discuss the potential molecular mechanisms underlying Nrf2-mediated dichotomy in the heart.

\section{Nrf2 SIGNALING}

Nrf2-related factor 2 is a member of the Cap " $n$ " Collar (CNC) family of basic leucine zipper (bZip) transcription factors that include nuclear factor-erythroid factor 2 (NFE2), Nrf1-3, and broad-complex, tramtrack, and bric-a-brac (BTB) and CNC homolog 1 (Bach 1) and Bach 2. The differences between these transcription factors have been broadly reviewed (Li et al., 2009a; Maher and Yamamoto, 2010). Concisely, NF-E2 and Nrf1-3 act as transcriptional activators, whereas Bach 1 and 2 serve as transcriptional repressors. The expression of Nrf proteins is ubiquitous in the body. Nrf1 plays a role in controlling the basal gene expression level of some cytoprotective enzymes but does not regulate their inducible expression. Although marginal, Nrf3 is capable of regulating the gene expression of phase 2 enzymes. In contrast, Nrf2 binds to a cis-acting enhancer with a core nucleotide sequence of $5^{\prime}$-RTGACNNNGC-3', that is also known as the antioxidant response element (ARE), or the electrophile response element (EpRE), to control the basal and inducible expression of over 1000 genes that can be clustered into several groups with distinct functions, including antioxidative defense, detoxification, inflammatory responses, transcription factors, proteasomal and autophagic degradation, and metabolism (Hayes and Dinkova-Kostova, 2014; Cui et al., 2016; Kopacz et al., 2020). Thus, Nrf2 is multifunctional with cellular functions ranging from antioxidative defense to protein quality control to metabolism regulation.
Structural features of vertebrate Nrf2 have been revised and updated in detail (Hayes and Dinkova-Kostova, 2014; Silva-Islas and Maldonado, 2018; Kopacz et al., 2020). Briefly, Nrf2 has 7 Nrf2-erythroid-derived CNC homology (ECH; Neh) domains (Neh1-7), which are critical for its activity or its repression (Figure 1A). The amino-terminal Neh2 via the DLG and ETGE motifs binds the double glycine repeat (DGR) domain of Kelchlike ECH associating protein 1 (Keap1), a negative regulator of Nrf2. A nuclear localization signal (NLS) sequence is localized in this domain. The Neh6 is another degron region, a portion of a protein important for its degradation, which via the DSGIS and DSAPGS motifs, recruits the dimeric $\beta$-transducin repeatcontaining protein $(\beta$-TrCP), a substrate adaptor for the S-phase kinase 1 (Skp1)-Cullin 1 (Cul1)-Ring box protein 1 (Rbx1) core E3 ligase (i.e., $\mathrm{SCF}^{\beta-\mathrm{TrCP}}$ ). The Neh6 is important for Nrf2 degradation in stressed cells independent of Keap1. The carboxylterminal Neh3 is necessary for transcriptional activation of Nrf2 by recruiting coactivator, chromo-ATPase/helicase DNA-binding protein $(\mathrm{CDH})$ 6. The Neh3 contains a second NLS sequence. The Neh1 adjacent to the Neh3 contains a bZip structure, which is critical for DNA binding and dimerization with other transcription factors. A nuclear export signal (NES) sequence is localized in the Neh1. The Neh4 and Neh5 are two independent transactivation domains that interact with cAMP response element-binding protein (CREB)-binding protein (CBP) and/or receptor-associated coactivator 3 (RAC3). The Neh7 mediates repression of Nrf2 by physical interaction with retinoid $\mathrm{X}$ receptor alpha $(\mathrm{RXR} \alpha)$.

Nrf2-related factor 2 is a short-lived protein with a halflife less than $20 \mathrm{~min}$ in the cell (Li et al., 2009a; Chen and Maltagliati, 2018). The expression and activity of Nrf2 are tightly regulated at multiple levels, which have been recently reviewed (Hayes and Dinkova-Kostova, 2014; Chen and Maltagliati, 2018; Silva-Islas and Maldonado, 2018; Kopacz et al., 2020). Generally, the transcription of Nrf2 is activated by itself and other transcription factors, including the aryl hydrocarbon receptor (AhR), peroxisome proliferator-activated receptor (PPAR) $\alpha$ or PPAR $\gamma$, nuclear factor $(\mathrm{NF})-\kappa \mathrm{B}(\mathrm{NF}-\kappa \mathrm{B})$, specificity protein 1 (Sp-1), p53, myocyte-specific enhancer factor 2 D (MEF2D), c-Jun, c-Myc, and breast cancer 1 (BRCA1). Epigenetic regulations, such as methylation of the Nrf2 promoter in $\mathrm{CpG}$ islands or $\mathrm{H} 3$ histone and acetylation of $\mathrm{H} 4$ histone, are also involved in Nrf2 transcriptional control. Moreover, Nrf2 synthesis can be downregulated by several miRNAs, including miR27a, miR-28, miR-34a, miR-93, miR-129-5p, miR142-5p, miR-144, miR-153, miR-155, miR-200c, miR-340-5p, miR-350a, miR-507, and miR-634 at the posttranscriptional level. Nevertheless, the protein stability and transcriptional activity of $\mathrm{Nrf} 2$ are mainly regulated by Keap1. Keap1 contains two major domains, BTB and DGR or Kelch, and three additional domains, the N-terminal region (NTR), the intervening region (IVR), and CTR (Figure 1A). The BTB is critical for Keap1 homodimerization and interaction with Cul3-Rbx1-E3 ligase complex while the Kelch binds to the DLG and ETGE motifs in Neh2 of Nrf2. Keap1 contains many cysteine residues sensing oxidative and/or electrophilic molecules in both BTB and IVR. Normally, Keap1 constantly targets Nrf2 for degradation. 


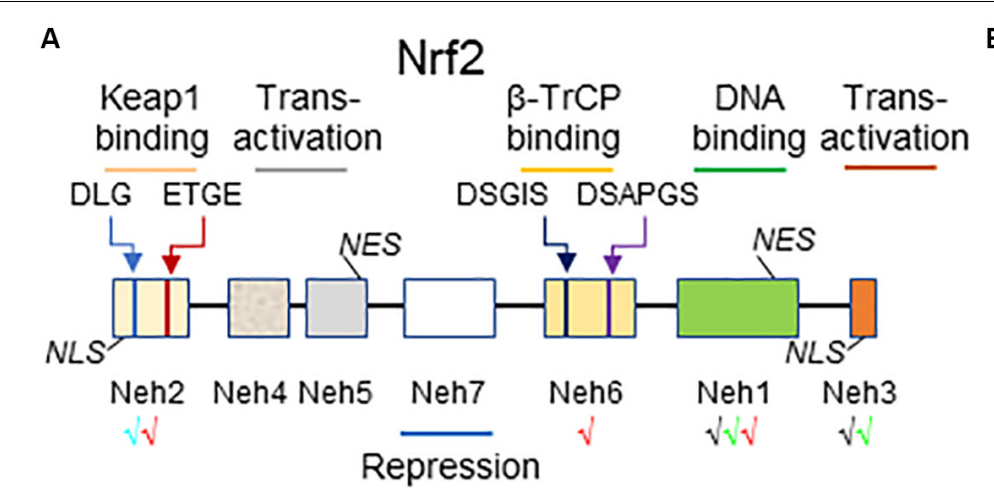

Acetylation ( $\sqrt{ }$ ); SUMOylation ( $)$; Ubiquitination ( $)$ : Phosphorylation $(\sqrt{ })$
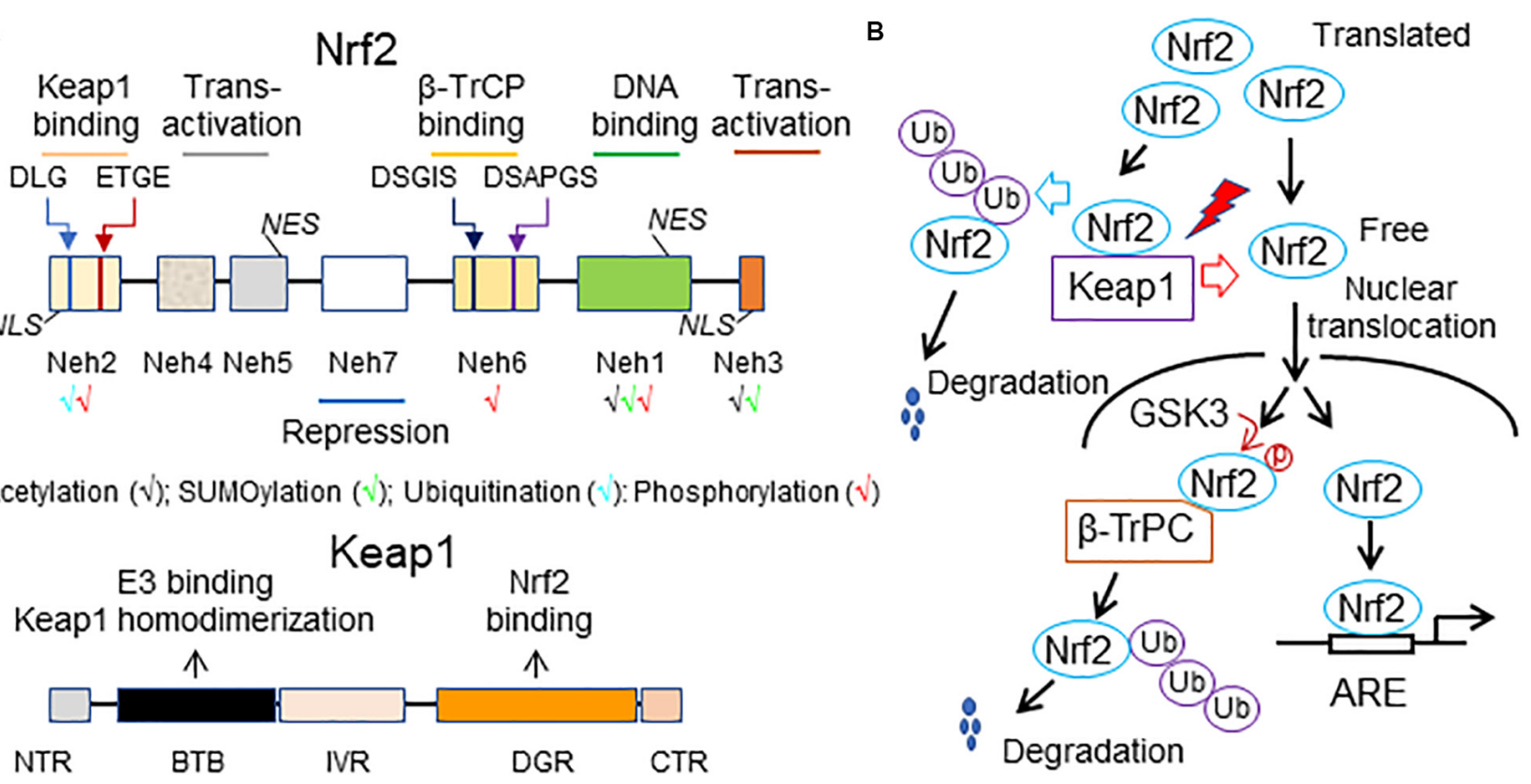

FIGURE 1 | A working model of Nrf2 signaling. (A) Structural features of Nrf2 and Keap1. The high-affinity ETGE and low-affinity DLG motifs in the Neh2 domain of Nrf2 are bound by the Kelch domain of Keap1 for Nrf2 ubiquitination and degradation. Neh, Nrf2-erythroid-derived CNC homology (ECH) domain; NES, nuclear export signal; $\beta$-TrCP, the dimeric $\beta$-transducin repeat-containing protein; NLS, nuclear localization signal; BTB, broad-complex, tramtrack and bric-a-brac domain; DGR or Kelch, double glycine repeat domain; NTR, the N-terminal region; IVR, intervening region; CTR, the carboxyl terminal region. (B) A scheme of Nrf2 activity regulation. Under homeostatic conditions, translated Nrf2 binds to Keap1, leading to ubiquitination of Nrf2 and subsequent proteasomal degradation of the ubiquitinated Nrf2. As a result, there is only a small amount of free Nrf2 translocating into the nucleus and activating the basal expression of Nrf2 target genes. Under stressed conditions, various stressors (red lighting symbol) interrupt the interaction of Keap1 with Nrf2, thus resulting in decreases in proteasomal degradation of Nrf2 and increases in de novo Nrf2 free of Keap1 binding. Accordingly, the nuclear translocation of Nrf2 and subsequent Nrf2-driven transcription are increased, thereby intensifying Nrf2-mediated defense against the stress-induced imbalance of redox status and damage in the cell. An alternative mechanism of proteasomal degradation of Nrf2 independent of Keap1 is mediated by glycogen synthase kinase 3 (GSK3) and $\beta$-TrCP. Under normal conditions, GSK3 is maintained in an inactive state due to its inhibition by AKT-mediated phosphorylation at its N-terminal pseudosubstrate domain. However, once AKT is inactivated, GSK3 phosphorylates Nrf2 at the Neh6 domain. This phosphorylation recruits $\beta$-TrCP and initiates $\beta$-TrCP-mediated proteasomal degradation of Nrf2. Ub, ubiquitin.

The current diagram of Keap1 and Nrf2 interaction for Nrf2 degradation is the "hinge and latch" model: The ETGE motif of Nrf2 acts as the "hinge" while the DIG motif of Nrf2 functions as the "latch." Nrf2 sequentially binds to first one of the Keap1 homodimers via the ETGE motif to form an "open" conformation, prior to the DLG motif being captured by the other Keap1 to form a "closed" conformation that enables Nrf2 ubiquitination by Clu3-Rbx1-E3 ligase for proteasomal degradation and subsequently release of free Keap1. The free or regenerated Keap1 bind to newly synthesized Nrf2 to start another cycle of the Nrf2 degradation. Only a small and steady amount of Nrf2 that is not sequestered by Keap1 for degradation translocate into the nucleus, contributing to the basal expression of ARE-driven genes (Figure 1B). Under stressed conditions, the stressors that are usually oxidative or electrophilic molecules react with the cysteine residues of Keap1 to cause conformational changes in Keap1, leading to downregulation of Keap1-mediated ubiquitination and degradation of Nrf2. However, such modulation of Keap1 does not result in release of Nrf2; instead, it stabilizes the Keap1-Nrf2 interaction that blocks Nrf2 ubiquitination, thereby saturating the cellular pool of the Keap1-E3 complex. As a result, de novo Nrf2 free of Keap1 binding that translocates into the nucleus is increased, thereby enhancing the transcription of Nrf2-driven genes. However, there is an alternative mechanism for proteasomal degradation of Nrf2 independent of Keap1, which is mediated by glycogen synthase kinase 3 (GSK3) and $\beta$-TrCP (Figure 1B). Under normal conditions, GSK3 is maintained in an inactive state due to its inhibition by AKT-mediated phosphorylation at its N-terminal pseudosubstrate domain. However, once AKT is inactive, GSK3 phosphorylates Nrf2 at the Neh6. This phosphorylation recruits $\beta$-TrCP and initiates $\beta$-TrCP-mediated proteasomal degradation of Nrf2. Usually, Keap1-mediated proteasomal degradation of Nrf2 occurs in the cytosol, whereas GSK3-mediated proteasomal degradation of Nrf2 happens in the nucleus. The relative importance of Keap1 and GSK3- $\beta$-TrCP in controlling the magnitude and duration of Nrf2 activation remains poorly understood.

It should be noted that not only GSK3, but also other kinases, such as AMP-activated protein kinase (AMPK) and mechanistic target of rapamycin complex 1 (mTORC1), directly or indirectly regulate Nrf2 stability and activity (Hayes and Dinkova-Kostova, 2014; Silva-Islas and Maldonado, 2018). In addition, ubiquitination of Nrf2 at the Neh2 can suppress Nrf2 activity, whereas acetylation of Nrf2 at the Neh1 and Neh3 may activate Nrf2 activity. SUMOylation of Nrf2 at the Neh1 and 
Neh3 may result in either Nrf2 activation or Nrf2 repression (Hayes and Dinkova-Kostova, 2014; Silva-Islas and Maldonado, 2018). Moreover, not only oxidative and electrophilic reactions, but also other types of posttranslational modifications of Keap1, such as ubiquitination and phosphorylation, appear to be critical for regulating Nrf2 activity, which has been comprehensively discussed in a recent review (Kopacz et al., 2020). However, the pathophysiological relevance of these findings in the heart remains to be investigated.

\section{Nrf2-MEDIATED CARDIAC PROTECTION}

Zhu et al. (2008) demonstrated for the first time an Nrf2dependent cytoprotection against oxidative and electrophilic stress in cardiomyocytes using neonatal mouse ventricular myocytes of Nrf2 knockout (KO) mice. He et al. subsequently documented that $\mathrm{KO}$ of $\mathrm{Nrf} 2$ enhances ROS production and exaggerates cell death in cultured adult cardiomyocytes in a setting of high glucose-induced oxidative stress ( $\mathrm{He}$ et al., 2009). While Sussan et al. (2009) found that global KO of Nrf2 enhances cigarette smoke-induced cardiac dysfunction in mice, we further demonstrated that the loss of Nrf2 function accelerates the transition from cardiac compensatory adaptation to heart failure in a setting of pressure overload ( $\mathrm{Li}$ et al., 2009 b). As we demonstrated that pharmacological activation of Nrf2 suppresses oxidative stress-dependent death in cardiac myocytes (Ichikawa et al., 2009; Li et al., 2010), Zhang et al. (2010) documented that Nrf2-deficient cardiomyocytes are more susceptible to 4-hydroxy-2-nonenal (4-HNE) challenge, and the cardiac protection of 4 -HNE pre-conditioning is dependent on Nrf2-operated antioxidant defense. Ashrafian et al. (2012) also showed that fumarate-induced suppression of ischemiareperfusion myocardial injury is wiped out in global Nrf2 $\mathrm{KO}$ mice, and Katsumata et al. revealed that Nrf2 is also essential for prostaglandin $\mathrm{D} 2\left(\mathrm{PGD}_{2}\right)$-mediated cardiac protection against ischemia-reperfusion injury in mice (Katsumata et al., 2014). We further demonstrated that conventionally cardiomyocyterestricted (CR) transgenic overexpression of Nrf2 protects against myocardial oxidative stress, cell death, fibrosis, hypertrophy, and dysfunction in a setting of sustained pressure overload induced by 4 weeks of transverse aortic arch constriction (TAC) in mice (Wang et al., 2014). Moreover, He et al. reported that global $\mathrm{KO}$ of Nrf2 could exaggerate cardiac oxidative stress, fibrosis, and apoptosis; contractility of cardiomyocytes; and death within 2 weeks after onset of type 1 diabetes induced by a single i.p. injection of $150 \mathrm{mg} / \mathrm{kg}$ streptozotocin (STZ) in mice (He and $\mathrm{Ma}, 2012$ ), and Gu et al. (2017) showed similar phenotypes in $\mathrm{Nrf} 2 \mathrm{KO}$ mice associated with type 2 diabetes, that is induced by 7 months of a high fat diet (HFD) containing $60 \% \mathrm{kcal}$ fat with a single i.p. injection of $100 \mathrm{mg} / \mathrm{kg} \mathrm{STZ}$ at 3 months. Collectively, these findings clearly demonstrate a cardioprotective role of $\mathrm{Nrf} 2$ in various pathological settings. Other studies regarding Nrf2-mediated cardiac protection and potential underlying mechanisms have been recently reviewed (Cui et al., 2016; Chen and Maltagliati, 2018; da Costa et al., 2019; Ge et al., 2019). Mechanistically, Nrf2 may activate antioxidant defense, regulate metabolism, and control autophagy and proteasome function, thereby contributing to cardiac protection.

\section{Nrf2-MEDIATED CARDIAC DAMAGE}

A Nrf2-mediated myocardial injury was first observed by Kannan et al. (2013) in aging cardiomyocyte-restricted human mutant CryAB transgenic (CR-hCryAB Tg) mice. Of note, CRhCryAB overexpression-induced death and cardiac dysfunction associated with aging were dramatically rescued by global KO of Nrf2. At the molecular level, the persistent activation of Nrf2-driven antioxidant gene expression toward a reductive stress has been proposed as a contributing mechanism to CRhCryAB Tg-induced cardiomyopathy (Kannan et al., 2013). While Allwood et al. found that CR transgenic overexpression of Ho1, an established downstream gene of Nrf2 in the heart (Li et al., 2009b), results in spontaneous development of heart failure at age of 1 year and exacerbates pressure overload-induced cardiomyopathy in mice (Allwood et al., 2014), we demonstrated that pathophysiological consequences of Nrf2 activation are linked to the functional integrity of autophagy in pressure overloaded mouse hearts (Qin et al., 2016). We have established that pressure overload via TAC initially results in an adaptive cardiac hypertrophy with preserved cardiac function (weeks 1-2) followed by maladaptive cardiac remodeling and dysfunction (weeks 2-4), which eventually causes heart failure in wild-type mice (Li et al., 2009b). Using this TAC model, we found that TAC-induced myocardial necrosis and death rate are increased in Nrf2 $\mathrm{KO}$ mice in a $\mathrm{C} 57 \mathrm{BL} / 6 \mathrm{~J}$ genetic background within first 2 weeks (Qin et al., 2016). These results underscore a critical role of Nrf2 in mediating cardiac protection during the initial stage of pressure overloadinduced cardiac adaptation. However, we found unexpectedly that Nrf2 KO attenuates cardiac hypertrophy and ameliorates progression of cardiac dysfunction by 8 weeks after TAC (Qin et al., 2016). These results reveal a mediator role of Nrf2 in pressure overload-induced cardiac maladaptive remodeling and dysfunction. A time course study of autophagy functional states in wild-type C57BL/6J mice showed that myocardial autophagy flux is intact at 2 weeks, suppressed at 4 weeks, and blocked at 8 weeks in the hearts after TAC (Qin et al., 2016). Since Nrf2 KO diminishes cardiac adaptation and leads to cardiac dysfunction at 2 weeks after TAC (Li et al., 2009b), when cardiac autophagy flux remains normal, it is most likely that Nrf2 activation is cardioprotective in pressure overloaded hearts when myocardial autophagy function is intact. Given that the Nrf2-mediated cardiac pathological hypertrophy and dysfunction are associated with impaired autophagy in the heart, it is conceivable that Nrf2 activation is detrimental to autophagyimpaired hearts. Indeed, genetic inhibition of autophagy, such as CR-Atg $5 \mathrm{KO}$, in combination with pharmacological kinase inhibitors, demonstrated that cardiac autophagy inhibition activates Fyn-operating Nrf2 nuclear export for degradation, thus enhancing Nrf2-driven transcription of angiotensinogen in cardiomyocytes, thereby leading to pathological activation of renin-angiotensin system in pressure overloaded hearts. These 
results are seemly contradictory to our findings that $\mathrm{Nrf} 2$ activation enhances autophagosome formation and autophagic degradation of protein aggregates, thereby protecting against 4-week TAC-induced cardiac maladaptive remodeling and dysfunction in FVB/N mice (Wang et al., 2014). However, it should be noted that Nrf2 does not regulate the expression of any autophagy-related genes, suggesting that Nrf2 may not directly activate autophagy but indirectly facilitates autophagy activation and degradation in cardiomyocytes (Wang et al., 2014). Given that genetic backgrounds have great impact on autophagy regulation in the heart (Moulis and Vindis, 2017), TAC induces myocardial autophagy inhibition at 4 weeks in C57BL/6J mice (Qin et al., 2016), but it may not induce the same phenotype at 4 weeks in $\mathrm{FVB} / \mathrm{N}$ mice. Although this notion remains to be clarified, previous studies have shown that the basal level of myocardial autophagy is much higher in $\mathrm{FVB} / \mathrm{N}$ mice $(\sim 1$-fold increases in autophagic flux by $6 \mathrm{~h}$ of chloroquine at a dose of $50 \mathrm{mg} / \mathrm{kg}$, i.p.) compared to C57BL/6J mice $(\sim 0.5$ fold increases in autophagic flux by $6 \mathrm{~h}$ of chloroquine at a dose of $50 \mathrm{mg} / \mathrm{kg}$, i.p.; Fernandez et al., 2018; Deng et al., 2020). Thus, a plausible explanation is that CR-Nrf2 Tg overexpressioninduced cardiac protection is mostly likely due to an intact state of myocardial autophagy by 4 weeks after TAC in FVB/N mice. Collectively, our findings suggest that autophagy impairment switches on Nrf2-mediated cardiac pathological remodeling and dysfunction. Interestingly, Bhide et al. (2018) documented that in a Drosophila model of laminopathy, laminopathy-associated agedependent cardiac dysfunction, could be rescued by knockdown of Nrf2, or enhancement of autophagy in the heart. These findings suggest that age-dependent autophagy deficiency may turn on Nrf2-mediated cardiac dysfunction in the Drosophila model of laminopathy. On the other hand, Erkens et al. (2018) reported that global $\mathrm{KO}$ of Nrf2 attenuates myocardial ischemia-reperfusion injury and dysfunction most likely due to

TABLE 1 | Nrf2 signaling cascade in mediating cardiac damage and dysfunction.

\begin{tabular}{|c|c|c|c|c|c|c|}
\hline Study & Nrf2 signaling & Animal model & Pathological setting & Intervention & Phenotype & $\begin{array}{l}\text { Proposed } \\
\text { mechanism }\end{array}$ \\
\hline $\begin{array}{l}\text { Kannan et al., } \\
2013\end{array}$ & Nrf2 & $\begin{array}{l}\text { CR-hCryAB Tg and } \\
\text { global Nrf2 KO in } \\
\text { C57BL/6J mice }\end{array}$ & Aging & None & $\begin{array}{l}\text { CR-hCryAB Tg-induced } \\
\text { cardiac accumulation of } \\
\text { protein aggregates and } \\
\text { reductive stress, } \\
\text { cardiomyopathy, and } \\
\text { heart failure are } \\
\text { rescued by additional } \\
\text { global KO of Nrf2 }\end{array}$ & $\begin{array}{l}\text { Nif2-mediated } \\
\text { reductive stress in the } \\
\text { heart }\end{array}$ \\
\hline $\begin{array}{l}\text { Allwood et al., } \\
2014\end{array}$ & $\mathrm{HO}-1$ & $\begin{array}{l}\text { CR-Ho1 Tg in FVB } \\
\text { mice }\end{array}$ & $\begin{array}{l}\text { Aging, Pressure } \\
\text { overload (PO), Excess } \\
\text { beta-adrenergic activity } \\
\text { (Isoproterenol infusion), }\end{array}$ & None & $\begin{array}{l}\text { CR-Ho1 Tg mice } \\
\text { develop spontaneous } \\
\text { heart failure at age of } \\
1 \text { year, and } \\
\text { exacerbated cardiac } \\
\text { dysfunction induced by } \\
\text { PO }\end{array}$ & $\begin{array}{l}\mathrm{HO}-1 \text {-mediated loss of } \\
\text { adaptive angiogenesis }\end{array}$ \\
\hline Qin et al., 2016 & Nrf2 & $\begin{array}{l}\text { Global Nrf2 KO in } \\
\text { C57BL/6J mice }\end{array}$ & Pressure overload (PO) & None & $\begin{array}{l}\text { Sustained PO leads to } \\
\text { cardiac autophagy } \\
\text { impairment and Nrf2 } \\
\text { activation; Nrf2 KO } \\
\text { attenuates progression } \\
\text { of cardiac pathological } \\
\text { remodeling and } \\
\text { dysfunction in PO } \\
\text { hearts. }\end{array}$ & $\begin{array}{l}\text { Sustained PO leads to } \\
\text { cardiac autophagy } \\
\text { impairment, which in } \\
\text { turn activates } \\
\text { Nrf2-driven } \\
\text { angiotensinogen } \\
\text { expression, thereby } \\
\text { contributing to } \\
\text { progression of cardiac } \\
\text { pathological remodeling } \\
\text { and dysfunction }\end{array}$ \\
\hline $\begin{array}{l}\text { Erkens et al., } \\
2018\end{array}$ & Nrf2 & $\begin{array}{l}\text { Global Nrf2 KO in } \\
\text { C57BL/6J mice }\end{array}$ & $\begin{array}{l}30 \text { min myocardial } \\
\text { ischemia followed by } \\
24 \mathrm{~h} \text { of reperfusion } \\
\text { in vivo }\end{array}$ & $\begin{array}{l}\text { Infusion of NOS } \\
\text { inhibitor } \\
\text { S-ethylisothiourea } \\
\text { hydrobromide } \\
\text { (ETU) }\end{array}$ & $\begin{array}{l}\text { Nrf2KO attenuates } \\
\text { myocardial } \\
\text { ischemia/reperfusion } \\
\text { injury and dysfunction }\end{array}$ & $\begin{array}{l}\text { Nrf2KO upregulates } \\
\text { cardiac NO }\end{array}$ \\
\hline $\begin{array}{l}\text { Bhide et al., } \\
2018\end{array}$ & Nrf2 & $\begin{array}{l}\text { CR-LamC-R205W and } \\
\text { G489V Tg and CncC } \\
\text { (Nrf2) RNAi in } \\
\text { Drosophila }\end{array}$ & Aging, Laminopathy & None & $\begin{array}{l}\text { CR-LamC mutant } \\
\text { Tg-induced } \\
\text { age-dependent cardiac } \\
\text { dysfunction is rescued } \\
\text { by knockdown of Nrf2 }\end{array}$ & $\begin{array}{l}\text { Autophagy impairment; } \\
\text { Nrf2 persistent } \\
\text { activation leading to } \\
\text { disruption of redox } \\
\text { homeostasis, defective } \\
\text { mitochondria, } \\
\text { dysregulation of energy } \\
\text { homeostasis and } \\
\text { energy sensor }\end{array}$ \\
\hline
\end{tabular}


an increase in cardiac nitric oxide (NO) production in mice, revealing a detrimental effect of $\mathrm{Nrf2}$-mediated suppression of NO production to the heart. Taken together, these genetic studies have clearly demonstrated a detrimental role of $\mathrm{Nrf} 2$ in the heart and Nrf2-mediated myocardial damage is likely occurring during the disease progression (Table 1). Since cardiac function is normal in CR-Nrf2 Tg mice at the age of $\sim 3$ months (Wang et al., 2014), it is highly possible that additional factors are required to turn on the Nrf2-mediated reductive stress causing cardiomyopathy. Although the precise mechanisms activating Nrf2-mediated cardiac damage are unclear, myocardial autophagy inhibition may be one of the critical triggers. However, the downstream signaling of Nrf2-mediated myocardial damage remains to be dissected.

\section{FINAL REMARK}

It is evident that Nrf2 could either protect against or exacerbate cardiac damage and dysfunction depending on the pathological nature of disease settings. Our data shows that Nrf2 is crucial for cardiac adaptation when cardiac autophagy is normal while exaggerating cardiac pathological decompensation when myocardial autophagy is impaired in pressure-overloaded hearts (Qin et al., 2016). Notably, autophagy inhibition is a contributory mechanism of protein aggregate-induced cardiomyopathies in aging CR-hCryAB Tg mice (Bhuiyan et al., 2013), sustained pressure overloaded mice (Qin et al., 2016), myocardial ischemiareperfusion mice (Ma et al., 2012), and aging Drosophila with laminopathy (Bhide et al., 2018). Therefore, it is likely that autophagy inhibition is essential for activating Nrf2-mediated cardiac damage toward heart failure. These results raise a concern regarding the potential activation of Nrf2-mediated cardiac damage in the clinical therapies when treated subjects are compounded with diabetic, hypertensive, and ischemic cardiomyopathies, all of which likely have myocardial autophagy inhibition (Wang and Cui, 2017).

Notably, the clinical phase III trial testing the therapeutic effect of Bardoxolone methyl, a potent Nrf2 activator, on chronic renal disease associated with type 2 diabetes was terminated because of an increased rate of cardiovascular events, including heart failure and deaths (de Zeeuw et al., 2013). The underlying mechanism remains to be determined. Given that autophagy inhibition also occurs in diabetic hearts (Ouyang et al., 2014; Kobayashi and Liang, 2015), it is intriguing whether the "dark" side of Nrf2 activation contributes to the failure of the Bardoxolone methyl

\section{REFERENCES}

Ahmed, Z., and Tang, W. H. (2012). Pharmacologic strategies to target oxidative stress in heart failure. Curr. Heart Fail Rep. 9, 14-22. doi: 10.1007/s11897-0110081-5

Allwood, M. A., Kinobe, R. T., Ballantyne, L., Romanova, N., Melo, L. G., Ward, C. A., et al. (2014). Heme oxygenase-1 overexpression exacerbates heart failure with aging and pressure overload but is protective against isoproterenolinduced cardiomyopathy in mice. Cardiovasc. Pathol. 23, 231-237. doi: 10. 1016/j.carpath.2014.03.007 clinical trial. Nevertheless, the enthusiasm for activating Nrf2 as a novel approach to treat human disease, at least non-cardiac diseases, remains very high (Al-Sawaf et al., 2015; RobledinosAnton et al., 2019). Several clinical trials of a few Nrf2 activators for treating other types of disease, including Bardoxolone methyl, Omaveloxolone, dimethyl fumarate, ALKS-8700 (a fumarate acid ester), Oltipraz, Ursodiol, Sulforaphane, Sulforadex, and Curcumin are still actively ongoing (Robledinos-Anton et al., 2019). All these pharmacological Nrf2 activators are electrophilic compounds that could covalently modify cysteine residues in Keap1 by oxidation or alkylation to cause conformational changes in Keap1, leading to inhibition of Keap1-mediated degradation of $\mathrm{Nrf} 2$, thus increasing the amount of newly synthesized and free Nrf2 and consequent enhancement of Nrf2operating transcription (Robledinos-Anton et al., 2019). Thus, these so-called "Nrf2 activators" are actually "Keap1 inhibitors." Recent studies have revealed that Keap1 is not limited to control Nrf2 activity, but also required for S-nitrosation, proteostasis, mitochondria homeostasis, cytoskeleton regulation, and cell cycle progression (Kopacz et al., 2020). However, the impact of these Nrf2 activators or Keap1 inhibitors on the Keap1-mediated actions beyond inactivation of Nrf2 remains largely unknown.

Taken together, further investigation of molecular mechanisms of Nrf2-mediated myocardial damage, such as autophagy-mediated control of Nrf2 signaling in the heart, will lead to a better understanding of Nrf2-medicated dichotomy in the pathogenesis of cardiomyopathies toward heart failure. The off-target effects of Nrf2 activating compounds (Keap1 inhibitors) in the heart, particularly the possibility of interrupting Keap1 functions independent of Nrf2 degradation, have to be characterized. As a result, the outcome will provide novel insight into the development of new effective approaches to target Nrf2 signaling for the treatment of cardiac and non-cardiac diseases.

\section{AUTHOR CONTRIBUTIONS}

$\mathrm{HZ}$ and RM wrote the draft. TC provided research funds and finalized the manuscript. All authors contributed to the article and approved the submitted version.

\section{FUNDING}

This work was supported by the National Institute of Health (R01 HL131667) and American Diabetes Association (1-16-IBS-059).

Al-Sawaf, O., Clarner, T., Fragoulis, A., Kan, Y. W., Pufe, T., Streetz, K., et al. (2015). Nrf2 in health and disease: current and future clinical implications. Clin. Sci. 129, 989-999. doi: 10.1042/cs20150436

Ashrafian, H., Czibik, G., Bellahcene, M., Aksentijevic, D., Smith, A. C., Mitchell, S. J., et al. (2012). Fumarate is cardioprotective via activation of the Nrf2 antioxidant pathway. Cell Metab. 15, 361-371.

Bhide, S., Trujillo, A. S., O’Connor, M. T., Young, G. H., Cryderman, D. E., Chandran, S., et al. (2018). Increasing autophagy and blocking Nrf2 suppress laminopathy-induced age-dependent cardiac dysfunction and shortened lifespan. Aging Cell 17, e12747. doi: 10.1111/acel.12747 
Bhuiyan, M. S., Pattison, J. S., Osinska, H., James, J., Gulick, J., McLendon, P. M., et al. (2013). Enhanced autophagy ameliorates cardiac proteinopathy. J. Clin. Invest. 123, 5284-5297. doi: 10.1172/jci70877

Chen, Q. M., and Maltagliati, A. J. (2018). Nrf2 at the heart of oxidative stress and cardiac protection. Physiol. Genomics 50, 77-97. doi: 10.1152/physiolgenomics. 00041.2017

Cohn, J. N., Ferrari, R., and Sharpe, N. (2000). Cardiac remodeling-concepts and clinical implications: a consensus paper from an international forum on cardiac remodeling. Behalf of an International Forum on Cardiac Remodeling. J. Am. Coll. Cardiol. 35, 569-582. doi: 10.1016/s0735-1097(99)00 630-0

Cui, T., Lai, Y., Janicki, J. S., and Wang, X. (2016). Nuclear factor erythroid2 related factor 2 (Nrf2)-mediated protein quality control in cardiomyocytes. Front. Biosci. 21:192-202. doi: 10.2741/4384

da Costa, R. M., Rodrigues, D., Pereira, C. A., Silva, J. F., Alves, J. V., Lobato, N. S., et al. (2019). Nrf2 as a potential mediator of cardiovascular risk in metabolic diseases. Front. Pharmacol. 10:382. doi: 10.3389/fphar.2019. 00382

de Zeeuw, D., Akizawa, T., Audhya, P., Bakris, G. L., Chin, M., Christ-Schmidt, H., et al. (2013). Bardoxolone methyl in type 2 diabetes and stage 4 chronic kidney disease. N. Engl. J. Med. 369, 2492-2503.

Deng, S., Essandoh, K., Wang, X., Li, Y., Huang, W., Chen, J., et al. (2020). Tsg101 positively regulates P62-Keap1-Nrf2 pathway to protect hearts against oxidative damage. Redox Biol. 32:101453. doi: 10.1016/j.redox.2020.10 1453

Erkens, R., Suvorava, T., Sutton, T. R., Fernandez, B. O., Mikus-Lelinska, M., Barbarino, F., et al. (2018). Nrf2 deficiency unmasks the significance of nitric oxide synthase activity for cardioprotection. Oxid. Med. Cell Longev. 2018:8309698.

Fernandez, A. F., Sebti, S., Wei, Y., Zou, Z., Shi, M., McMillan, K. L., et al. (2018). Disruption of the beclin 1-BCL2 autophagy regulatory complex promotes longevity in mice. Nature 558, 136-140. doi: 10.1038/s41586-0180162-7

Ge, Z. D., Lian, Q., Mao, X., and Xia, Z. (2019). Current status and challenges of NRF2 as a potential therapeutic target for diabetic cardiomyopathy. Int. Heart J. 60, 512-520. doi: 10.1536/ihj.18-476

Gu, J., Cheng, Y., Wu, H., Kong, L., Wang, S., Xu, Z., et al. (2017). Metallothionein is downstream of $\mathrm{Nrf} 2$ and partially mediates sulforaphane prevention of diabetic cardiomyopathy. Diabetes 66, 529-542. doi: $10.2337 / \mathrm{db} 15-$ 1274

Hayes, J. D., and Dinkova-Kostova, A. T. (2014). The Nrf2 regulatory network provides an interface between redox and intermediary metabolism. Trends Biochem. Sci. 39, 199-218. doi: 10.1016/j.tibs.2014.02.002

He, X., Kan, H., Cai, L., and Ma, Q. (2009). Nrf2 is critical in defense against high glucose-induced oxidative damage in cardiomyocytes. J. Mol. Cell Cardiol. 46, 47-58. doi: 10.1016/j.yjmcc.2008.10.007

He, X., and Ma, Q. (2012). Disruption of Nrf2 synergizes with high glucose to cause heightened myocardial oxidative stress and severe Cardiomyopathy in diabetic mice. J. Diabetes Metab. 7:002.

Heusch, G., Libby, P., Gersh, B., Yellon, D., Bohm, M., Lopaschuk, G., et al. (2014). Cardiovascular remodelling in coronary artery disease and heart failure. Lancet 383, 1933-1943. doi: 10.1016/s0140-6736(14)60107-0

Hunt, S. A., Abraham, W. T., Chin, M. H., Feldman, A. M., Francis, G. S., Ganiats, T. G., et al. (2005). ACC/AHA 2005 Guideline Update for the Diagnosis and Management of Chronic Heart Failure in the Adult: a report of the American College of Cardiology/American Heart Association Task Force on Practice Guidelines (Writing Committee to Update the 2001 Guidelines for the Evaluation and Management of Heart Failure): developed in collaboration with the American College of Chest Physicians and the International Society for Heart and Lung Transplantation: endorsed by the Heart Rhythm Society. Circulation 112, e154-e235.

Ichikawa, T., Li, J., Meyer, C. J., Janicki, J. S., Hannink, M., and Cui, T. (2009). Dihydro-CDDO-trifluoroethyl amide (dh404), a novel Nrf2 activator, suppresses oxidative stress in cardiomyocytes. PLoS One 4:e8391. doi: 10.1371/ journal.pone.0008391

Kannan, S., Muthusamy, V. R., Whitehead, K. J., Wang, L., Gomes, A. V., Litwin, S. E., et al. (2013). Nrf2 deficiency prevents reductive stress-induced hypertrophic cardiomyopathy. Cardiovasc. Res. 100, 63-73. doi: 10.1093/cvr/ cvt150

Katsumata, Y., Shinmura, K., Sugiura, Y., Tohyama, S., Matsuhashi, T., Ito, H., et al. (2014). Endogenous prostaglandin D2 and its metabolites protect the heart against ischemia-reperfusion injury by activating Nrf2. Hypertension 63, 80-87. doi: 10.1161/hypertensionaha.113.01639

Kobayashi, S., and Liang, Q. (2015). Autophagy and mitophagy in diabetic cardiomyopathy. Biochim. Biophys. Acta 1852, 252-261. doi: 10.1016/j.bbadis. 2014.05.020

Kopacz, A., Kloska, D., Forman, H. J., Jozkowicz, A., and Grochot-Przeczek, A. (2020). Beyond repression of Nrf2: an update on Keap1. Free Radic. Biol. Med. doi: 10.1016/j.freeradbiomed.2020.03.023 [Online ahead of print].

Li, J., Ichikawa, T., Janicki, J. S., and Cui, T. (2009a). Targeting the Nrf2 pathway against cardiovascular disease. Expert Opin. Ther. Targets 13, 785-794. doi: $10.1517 / 14728220903025762$

Li, J., Ichikawa, T., Villacorta, L., Janicki, J. S., Brower, G. L., Yamamoto, M., et al. (2009b). Nrf2 protects against maladaptive cardiac responses to hemodynamic stress. Arterioscler. Thromb. Vasc. Biol. 29, 1843-1850. doi: 10.1161/atvbaha. 109.189480

Li, J., Ichikawa, T., Jin, Y., Hofseth, L. J., Nagarkatti, P., Nagarkatti, M., et al. (2010). An essential role of Nrf2 in American ginseng-mediated anti-oxidative actions in cardiomyocytes. J. Ethnopharmacol. 130, 222-230. doi: 10.1016/j.jep.2010. 03.040

Ma, X., Liu, H., Foyil, S. R., Godar, R. J., Weinheimer, C. J., Hill, J. A., et al. (2012). Impaired autophagosome clearance contributes to cardiomyocyte death in ischemia/reperfusion injury. Circulation 125, 3170-3181. doi: 10.1161/ circulationaha.111.041814

Maher, J., and Yamamoto, M. (2010). The rise of antioxidant signaling-the evolution and hormetic actions of Nrf2. Toxicol. Appl. Pharmacol. 244, 4-15. doi: 10.1016/j.taap.2010.01.011

Moulis, M., and Vindis, C. (2017). Methods for measuring autophagy in mice. Cells 6:E14.

Ouyang, C., You, J., and Xie, Z. (2014). The interplay between autophagy and apoptosis in the diabetic heart. J. Mol. Cell Cardiol. 71, 71-80. doi: 10.1016/ j.yjmcc.2013.10.014

Qin, Q., Qu, C., Niu, T., Zang, H., Qi, L., Lyu, L., et al. (2016). Nrf2-mediated cardiac maladaptive remodeling and dysfunction in a setting of autophagy insufficiency. Hypertension 67, 107-117. doi: 10.1161/hypertensionaha.115. 06062

Robledinos-Anton, N., Fernandez-Gines, R., Manda, G., and Cuadrado, A. (2019). Activators and inhibitors of NRF2: a review of their potential for clinical development. Oxid. Med. Cell Longev. 2019:9372182.

Silva-Islas, C. A., and Maldonado, P. D. (2018). Canonical and non-canonical mechanisms of Nrf2 activation. Pharmacol. Res. 134, 92-99. doi: 10.1016/j.phrs. 2018.06.013

Sussan, T. E., Rangasamy, T., Blake, D. J., Malhotra, D., El-Haddad, H., Bedja, D., et al. (2009). Targeting Nrf2 with the triterpenoid CDDO-imidazolide attenuates cigarette smoke-induced emphysema and cardiac dysfunction in mice. Proc. Natl. Acad. Sci. U.S.A. 106, 250-255. doi: 10.1073/pnas.080433 3106

Swynghedauw, B. (1999). Molecular mechanisms of myocardial remodeling. Physiol. Rev. 79, 215-262. doi: 10.1152/physrev.1999.79.1.215

Tsutsui, H., Kinugawa, S., and Matsushima, S. (2011). Oxidative stress and heart failure. Am. J. Physiol. Heart Circ. Physiol. 301, H2181-H2190.

Virani, S. S., Alonso, A., Benjamin, E. J., Bittencourt, M. S., Callaway, C. W., Carson, A. P., et al. (2020). Heart disease and stroke statistics2020 update: a report from the american heart association. Circulation 141, e139-e596.

Wang, W., Li, S., Wang, H., Li, B., Shao, L., Lai, Y., et al. (2014). Nrf2 enhances myocardial clearance of toxic ubiquitinated proteins. J. Mol. Cell Cardiol. 72, 305-315. doi: 10.1016/j.yjmcc.2014.04.006

Wang, X., and Cui, T. (2017). Autophagy modulation: a potential therapeutic approach in cardiac hypertrophy. Am. J. Physiol. Heart Circ. Physiol. 313, H304-H319.

Yancy, C. W., Jessup, M., Bozkurt, B., Butler, J., Casey, D. E. Jr., Drazner, M. H., et al. (2013). 2013 ACCF/AHA guideline for the management of heart failure: executive summary: a report of the American College of Cardiology 
Foundation/American Heart Association Task Force on practice guidelines. Circulation 128, 1810-1852.

Zhang, Y., Sano, M., Shinmura, K., Tamaki, K., Katsumata, Y., Matsuhashi, T., et al. (2010). 4-hydroxy-2-nonenal protects against cardiac ischemia-reperfusion injury via the Nrf2-dependent pathway. J. Mol. Cell Cardiol. 49, 576-586. doi: 10.1016/j.yjmcc.2010.05.011

Zhu, H., Jia, Z., Misra, B. R., Zhang, L., Cao, Z., Yamamoto, M., et al. (2008). Nuclear factor E2-related factor 2-dependent myocardiac cytoprotection against oxidative and electrophilic stress. Cardiovasc. Toxicol. 8, 71-85. doi: 10.1007/s12012-008-9016-0
Conflict of Interest: The authors declare that the research was conducted in the absence of any commercial or financial relationships that could be construed as a potential conflict of interest.

Copyright $\odot 2020$ Zang, Mathew and Cui. This is an open-access article distributed under the terms of the Creative Commons Attribution License (CC BY). The use, distribution or reproduction in other forums is permitted, provided the original author(s) and the copyright owner(s) are credited and that the original publication in this journal is cited, in accordance with accepted academic practice. No use, distribution or reproduction is permitted which does not comply with these terms. 